\title{
Real-life results of switching from preserved to preservative-free artificial tears containing hyaluronate in patients with dry eye disease
}

This article was published in the following Dove Press journal:

Clinical Ophthalmology

\author{
Linda Nasser ${ }^{1}$ \\ Malgorzata Rozycka ${ }^{2}$ \\ Gabriela Gomez Rendon ${ }^{3}$ \\ Alejandro Navas ${ }^{4}$ \\ 'Institute for Prevention of Blindness \\ in Nuevo Leon, Monterrey, Mexico; \\ ${ }^{2}$ Théa Laboratories, Mexico \\ City, Mexico; ${ }^{3}$ Private practice; \\ ${ }^{4}$ Cornea department, Institute of \\ Ophthalmology, Conde de Valenciana, \\ Mexico City, Mexico
}

Background: Dry eye disease (DED) is a chronic, multifactorial disease of the ocular surface leading to discomfort, visual disturbance, and tear film instability, with potential damage to the ocular surface.

Aim: The aim of this study was to assess the clinical benefit of a switch from preserved to preservative-free artificial tears (ATs) containing hyaluronate in patients with DED.

Materials and methods: This is a nationwide, multicenter, noninterventional, and transversal observational survey.

Results: The mean age was $51.0 \pm 15.4$ years, ranging from 6 to 96 years. The majority $(61.4 \%)$ was female. The mean Ocular Surface Disease Index (OSDI) before the switch was surprisingly high at $56.0 \pm 23.5$, and $73.0 \%$ of the patients had superficial punctate keratitis (SPK). The mean duration of use of preserved ATs before the switch was 15.8 \pm 12.1 months. OSDI scores and the presence of SPK correlated with the patients' ages but were independent of the duration of treatment with the preserved AT. The patients using ATs containing "soft" or "vanishing" preservatives presented exactly the same clinical pattern (level of OSDI and frequency of SPK) as those using ATs containing classical preservatives such as benzalkonium chloride (BAK). After switching to preservative-free AT containing hyaluronate (Hyabak ${ }^{\circledR}$ ), the OSDI of $97.0 \%$ of the patients improved, decreasing from an average of 56.0 to an average of 28.2 , with $23 \%$ of patients reporting a normal value of OSDI. The SPK frequency as well improved dramatically, with a frequency of positive fluorescein staining dropping from $73 \%$ to $46.1 \%$ of patients. A total of $94.0 \%$ of the patients considered that they preferred being treated with the preservative-free AT.

Conclusion: In patients suffering from DED and treated with a preserved AT, switching to a preservative-free AT provides clinical benefit by decreasing the severity of DED and reducing the prevalence of SPK, even after only 3 weeks of daily use of the preservative-free AT.

Keywords: dry eye syndrome, ocular surface diseases, preservative-free artificial tears, benzalkonium chloride free

\section{Introduction}

Dry eye is a multifactorial disease of the ocular surface, resulting in discomfort, visual disturbance, and tear film instability, with potential damage to the ocular surface. Dry eye disease (DED) occurs along with an increased osmolality of the tear film and subacute inflammation of the ocular surface. ${ }^{1}$ Stress to the ocular surface, including environmental and genetic factors, infection, endogenous stress, and antigens are the main triggers. Proinflammatory cytokines, chemokines, and matrix metalloproteinases lead to the expansion of autoreactive T-helper cells, which infiltrate the ocular surface
Correspondence: Linda Nasser Instituto para la Prevención de la Ceguera en Nuevo León 64460, Avenida Hermosillo 3333, Mitras Centro, Monterrey, Nuevo León, Mexico

Tel +52 8I 83464134

Email ipc@clubdeleonesdemonterrey.com 
and lacrimal gland, resulting in a vicious circle of damage to the ocular surface and inflammation..$^{2-4}$

DED affects $5 \%-34 \%$ of people globally, and its prevalence increases with age..$^{5-10}$ The large differences in prevalence are due to variations in study populations, differences in geographical, and differences in method and, until the middle of 2007, variations in the definition of the disease. ${ }^{4}$

The most common clinical tools used to evaluate the severity are the Ocular Surface Disease Index (OSDI) and fluorescein testing of the ocular surface to detect superficial punctate keratitis (SPK), frequently observed in patients with DED. ${ }^{1,11}$

A wide range of management options exists for DED, including artificial tears (ATs), anti-inflammatory agents, immunomodulators, occlusive devices, and environmental modifiers. ${ }^{12}$ To date, the most common treatment of DED consists in the regular use of ATs. A large number of products are available, combining in various proportions a limited number of ingredients such as glycerin, polyvinyl alcohol, propylene glycol, hydroxypropyl guar, carbomers, cellulose derivatives, and sodium hyaluronate. The ATs can be presented in low, medium, or high viscosity (gels) preparations, but no relation between viscosity and efficacy has been established yet.

Although ATs are considered as a very common and widely used treatment, no large, randomized, controlled studies have been carried out to evaluate the many types of ATs available. ${ }^{4}$ However, small, randomized studies have shown that ATs increase the tear film stability, reduce ocular surface stress, improve contrast sensitivity and the optical quality of the surface, and increase the patient's quality of life. ${ }^{4,13-18}$

Most of the ATs contain preservatives in order to maintain the sterility of eye drops in multidose containers. The preservatives act in a totally unspecific manner as a detergent or by oxidative mechanisms and thereby cause damage not only to contaminating bacteria and other microorganisms but also to the cells of the ocular surface. It has also been demonstrated that they also affect the contact lenses' physical properties, the trabecular meshwork, and the retina. Benzalkonium chloride (BAK) is the most commonly used preservative in ophthalmology, even though it is considered as the most toxic. More recently, some companies have developed so-called "soft" or "vanishing" preservatives, as Purite or Polyquad (Allergan Inc., Irvine, CA, USA), supposed to be less toxic. Unfortunately, several recent publications have demonstrated toxic and inflammatory effects not less than BAK on the ocular surface. ${ }^{19,20}$ Finally, it appears that the only solution to avoid the undesired effects of preservatives is the use of preservative-free ATs, ie, ATs in vials that can guarantee the sterility of the content despite the absence of preservative in the solution. The use of preservative-free ATs, when available, is highly recommended, including in the last version of the Dry Eye Workshop Study (DEWS), ${ }^{1}$ in order to limit the noxious side effects linked to the preservatives. The aim of the present survey was to assess the clinical benefits for the patients with DED switching from a preserved AT to a preservativefree AT, namely Hyabak ${ }^{\circledR}$, marketed by Laboratorios Théa (Mexico City, Mexico), containing sodium hyaluronate.

\section{Materials and methods}

This has been a nationwide, multicenter, noninterventional, and transversal observational survey conducted between April and September 2016 by 45 ophthalmologists at 41 study sites in Mexico. Written informed consent was obtained for all cases; in case of underage patients, written informed consent was also obtained from a parent or guardian of the patient. The study protocol was approved by ethics committee (Comité de Ética en Investigación Grupo de Evaluación sobre Conservadores en Oftalmologia). It complied with all local legal and ethical requirements for the conduct of observational surveys, and it was performed in accordance with the Declaration of Helsinki.

A total of $>1,200$ male and female patients of any age having used for at least 6 months preserved ATs to treat their DED have been recruited. The survey planned for the following two visits:

- At baseline visit, the physicians had to collect information about the age and gender of the patient, the preserved AT currently used and its duration of use, and the number of instillations per day. The clinical situation of the patients before treatment switch has also been evaluated, through OSDI scoring and corneal staining with fluorescein. Investigators then prescribed the preservative-free AT.

- After a period of at least 3 weeks of treatment with the preservative-free AT, a second and identical clinical evaluation has been performed. In addition, at the end of this follow-up visit, the patients assessed their global satisfaction with the preservative-free AT.

Safety data have not been collected. Spontaneously reported related adverse events have been managed through postmarketing pharmacovigilance.

Statistical analyses for demographic and baseline data were descriptive. No corrections for multiple testing have been made. The mean OSDI scores before and after the treatment switch have been compared through a paired $t$-test, whereas the frequencies of detected SPK before and after the treatment 
switch have been compared through the McNemar test. Analyses of OSDI scores have used linear regression models, and SPK analyses have used logistic regression models. Evaluations prior to the treatment switch have been based on univariate models. Postswitch evaluations have been adjusted for values prior to switching. A significance level of 0.05 was applied. The $\mathrm{R}$ software (Comprehensive R Archive Network [CRAN]) has been used to analyze the results.

\section{Results}

\section{Demographic data}

Overall, data from 1,249 patients have been analyzed, the majority being female patients $(61.3 \%)$. The mean age was $51.0 \pm 15.4$ years, ranging from 6 to 96 years.

The mean duration of treatment with a preserved AT before treatment switch was 15.8 months (between 6 and 120 months). Complete demographics and baseline data are presented in Table 1.

The majority of patients used soft or vanishing preservatives, as Polyquad or Purite. The nature and prevalence of the preservative contained in the ATs used before treatment switch are presented in Figure 1. Figure 1 shows the data of OSDI score according to each preservative used before treatment switch to preservative-free AT.

\section{Clinical assessments before treatment switch}

Globally, according to their OSDI score, $81 \%$ of the patients had severe DED, $6.5 \%$ of the patients had moderate DED, and $9.9 \%$ of the patients had mild DED; $2.6 \%$ of the patients did not report DED.

A positive fluorescein staining, showing a SPK, has been observed in $73.0 \%$ of the patients; details for incidences per preservative are provided in Figure 2.

OSDI scores were significantly higher, and positive fluorescein staining was significantly more frequent (both $P<0.0001)$ in older adults $(0.42,95 \%$ CI $[0.34,0.50]$ and $1.03,95 \%$ CI $[1.02,1.04]$, respectively). Conversely, there was no relationship between the gender and duration of treatment and the OSDI scores or the positivity of the corneal staining.

The patients with the higher OSDI score were also those with a positive fluorescein staining $(15.61,95 \%$ CI [12.79, 18.44], $P<0.0001)$.

Regardless the preservative, the duration of treatment had no impact on OSDI scores $(0.06,95 \%$ CI $[-0.05,0.17]$, $P=0.2882)$ or on positive fluorescein staining $(1.00,95 \% \mathrm{CI}$ $[0.99,1.01], P=0.4003)$. There was no difference in OSDI
Table I Demographic and baseline disease data

\begin{tabular}{|c|c|}
\hline Total number of patients $(\mathrm{N})$ & 1,249 \\
\hline \multicolumn{2}{|l|}{ Age (years) } \\
\hline Mean \pm SD & $51.0 \pm 15.4$ \\
\hline Range & $6.0-96.0$ \\
\hline \multicolumn{2}{|l|}{ Gender, n (\%) } \\
\hline Female & $766(6 I .3)$ \\
\hline Male & $483(38.7)$ \\
\hline \multicolumn{2}{|l|}{ Preserved artificial tears } \\
\hline \multicolumn{2}{|l|}{ Duration (months) } \\
\hline Mean \pm SD & $15.8 \pm \mid 2.1$ \\
\hline Range & $6.0-120.0$ \\
\hline \multicolumn{2}{|l|}{ Daily instillations (n) } \\
\hline Median & 4 \\
\hline Range & $1-14$ \\
\hline \multicolumn{2}{|l|}{ Type of preservative, $n(\%)$} \\
\hline Traditional preservatives & $517(45.9)$ \\
\hline Soft preservatives & $607(54.1)$ \\
\hline Missing & 125 \\
\hline \multicolumn{2}{|l|}{ Preservative, n (\%) } \\
\hline BAK & $331(29.3)$ \\
\hline BAK/cetrimide & $4(0.4)$ \\
\hline Cetrimide & $133(11.7)$ \\
\hline Stabilized chloride peroxide & $6(0.5)$ \\
\hline Polyquaternium-I & $256(22.8)$ \\
\hline Stabilized oxychloro complex & $35 I(3 I .0)$ \\
\hline Thiomersal & $47(4.1)$ \\
\hline Others & $2(0.2)$ \\
\hline Mixed (including four BAK/cetrimide and two others) & 119 \\
\hline \multicolumn{2}{|l|}{ OSDI } \\
\hline Mean \pm SD & $56.0 \pm 23.5$ \\
\hline Range & $6.3-100$ \\
\hline \multicolumn{2}{|l|}{ SPK, n (\%) } \\
\hline No & $334(27.0)$ \\
\hline Yes & $898(73.0)$ \\
\hline Missing & 17 \\
\hline
\end{tabular}

Abbreviations: BAK, benzalkonium chloride; OSDI, Ocular Surface Disease Index; SPK, superficial punctate keratitis.

scores between patients using soft preservatives containing ATs and patients using BAK-containing traditional preservatives.

Compared to the patients using BAK-containing ATs, patients using ATs containing cetrimide (2.12, 95\% CI [1.27, $3.51], P<0.005)$ and Polyquad $(1.95,95 \%$ CI [1.31, 2.89], $P<0.0005)$ were associated with the development of SPK, while the association for patients using ATs containing Purite was similar $(1.07,95 \% \mathrm{CI}[0.77,1.49], P=0.6745547)$ to that for patients using BAK-containing ATs.

\section{Clinical assessments after the treatment switch OSDI values}

After the switch to the preservative-free AT, the mean OSDI score decreased from $56.0 \pm 23.5$ to $28.2 \pm 17.3$ (Figure 3); the 


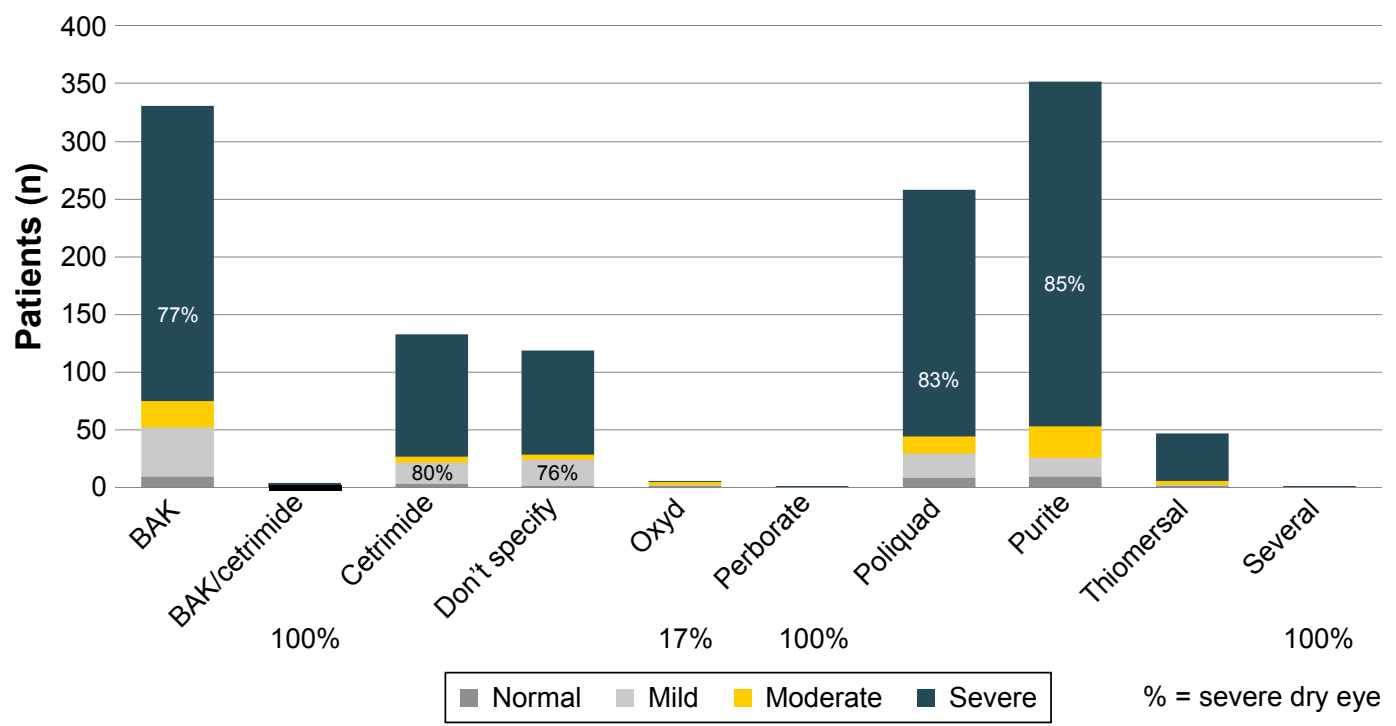

Figure I Type of preservative used and corresponding OSDI scores before treatment switch to preservative-free AT.

Abbreviations: AT, artificial tear; BAK, benzalkonium chloride; OSDI, Ocular Surface Disease Index; Oxyd, Stabilized Chlorite Complex; Purtite, Stabilized Oxychloro Complex.

difference is statistically significant $(P \leq 0.001)$. Overall, the OSDI of $97.0 \%$ of patients has decreased, with a parallel improvement in severe dry eye symptoms in $46 \%$ of the patients; $23 \%$ of the patients no longer had dry eyes (Figure 4 ).

Compared to patients using ATs containing traditional preservatives, such as BAK, patients using soft preservatives have significantly higher OSDI values after treatment switch, when correcting for potentially different OSDI values of patients before treatment switch (coefficient regression 0.45 [0.42, 0.49], $P<0001)$.

However, the duration of treatment does not influence the OSDI score before treatment switch; it is observed that patients who took a preserved AT for a longer duration before treatment switch have a significantly higher OSDI value after treatment switch $(-23.12[-25.16,-21.08], P<0.0001)$.

\section{Fluorescein staining}

After switching to the unpreserved AT, the frequency of SPK has decreased from $73 \%$ to $46.1 \%$ of patients $(P \leq 0.001$, Figure 5). As before treatment switch, the patients with the higher values of OSDI are also those with the highest association of SPK $(5.93$ [4.00, 7.85], $P<0.0001)$.

SPK after treatment switch correlated significantly with the patient's age $(1.02,95 \%$ CI $[1.01,1.03], P<0.0001)$, while there was no relationship between the probability to develop SPK and the gender.

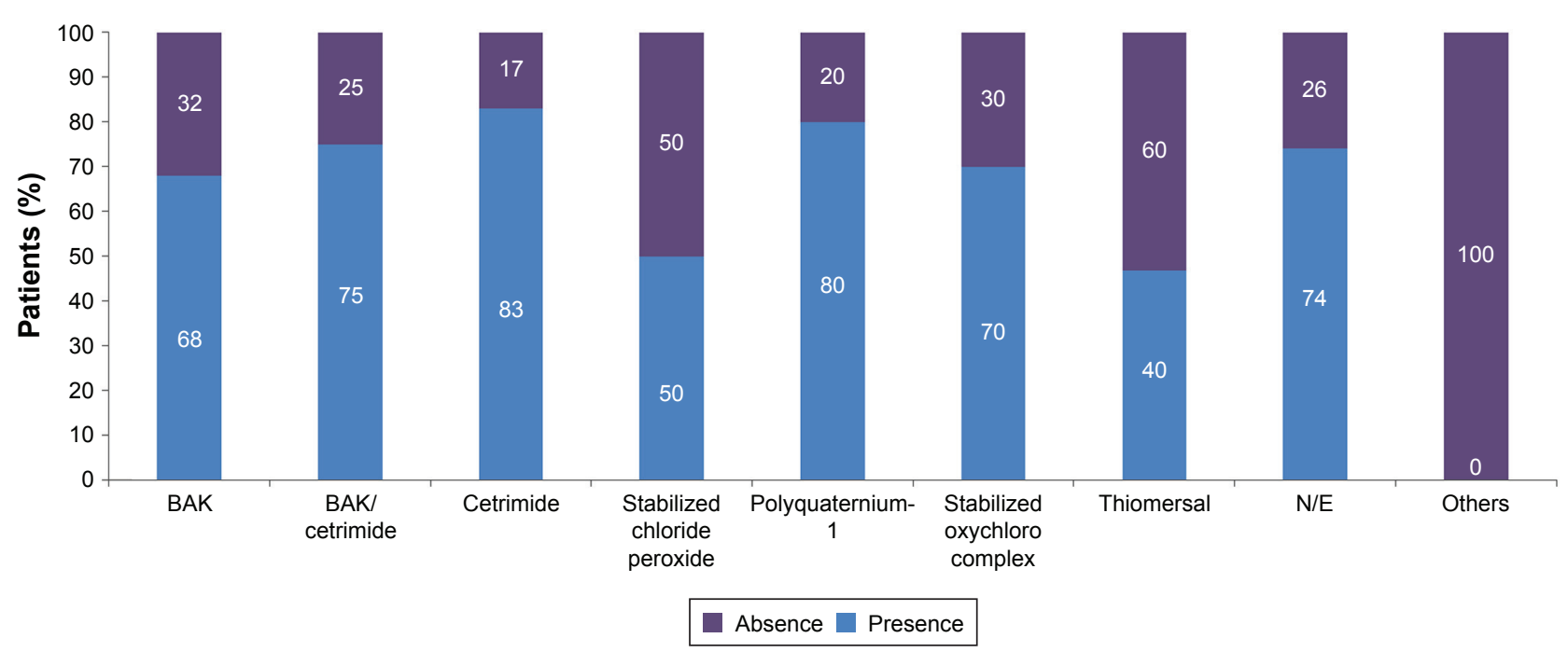

Figure 2 Percentage of patients with SPK at baseline according to preservatives.

Abbreviations: BAK, benzalkonium chloride; N/E, not evaluable; SPK, superficial punctate keratitis. 


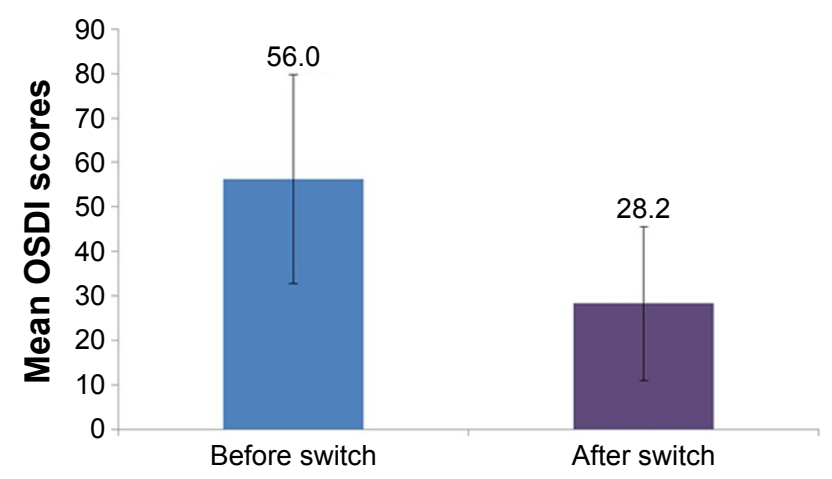

Figure 3 Mean OSDI score at baseline and follow-up visit. Note: The decrease was statistically significant $(P \leq 0.00 \mathrm{I})$. Abbreviation: OSDI, Ocular Surface Disease Index.

The presence of SPK after treatment switch and the duration of use of a preserved AT before treatment switch did not correlate.

When comparing the patients having used ATs containing traditional preservatives with those having used ATs containing soft preservatives, the association to develop SPK is significantly higher $(2.47,95 \%$ CI $[1.87,3.25], P<0.0001)$ in those patients having used ATs containing soft preservatives before the switch.

A total of $94.0 \%$ of the patients stated that they preferred being treated with the preservative-free AT than with the former, preserved one.

\section{Discussion}

On a country-specific level, these large-scale epidemiological data show that in Mexico, not only older adult patients suffer from DED but also patients of almost any age suffer from DED. The following two factors explained this distribution: the high level of pollution in the urban areas and the very low level of humidity during the dry season.

The present results confirm that a large majority of patients treated with preserved ATs still suffer from severe DED according to OSDI assessments and presented a SPK. There is no relationship between the duration of treatment with a preserved AT and the severity of the DED: even patients treated for a short time had developed signs and symptoms of severe DED. As expected, there is a clear relationship between the patient's age and increased OSDI scores and SPK when using preserved ATs.

Concerning preservatives, in order to avoid the damages caused by the traditional benzalkonium chloride (BAK), cetrimide or thiomerosal, some companies have developed so-called soft or vanishing preservatives, named Polyquad or Purite. It is therefore interesting to analyze, in real-life conditions, whether this new generation of preservatives is less harmful for the ocular surface than the former one. The results are surprising:

- Patients using soft preservatives had significantly $(P<0.0001)$ higher OSDI scores after tretament switch. This correlation is established as $45 \%$. This correlation does not exist before treatment switch.

- Patients using soft preservatives had a 2.47 times higher chance to develop SPK after treatment switch $(P<0.0001)$ than those using traditional preservatives. Also, in the case of SPK, this correlation before treatment switch does not reach statistical significance (OR 1.31, $P=0.0434)$.
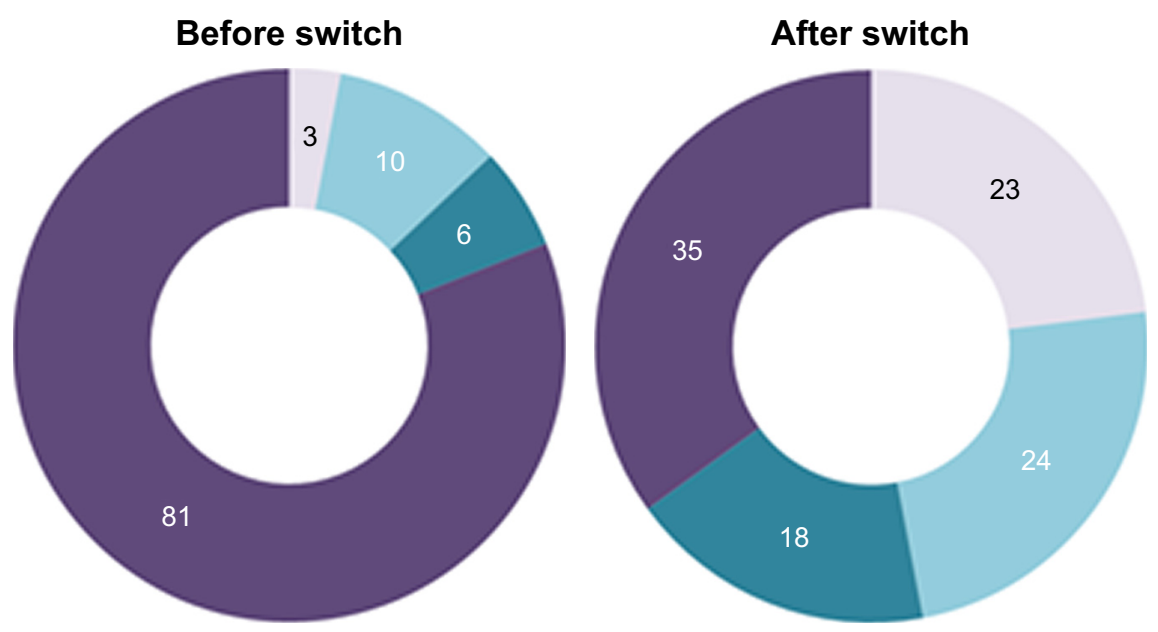

Normal Mild $\square$ Moderate $\square$ Severe

Figure 4 Percentage distribution of OSDI severity in patients before and after treatment switch.

Abbreviation: OSDI, Ocular Surface Disease Index. 


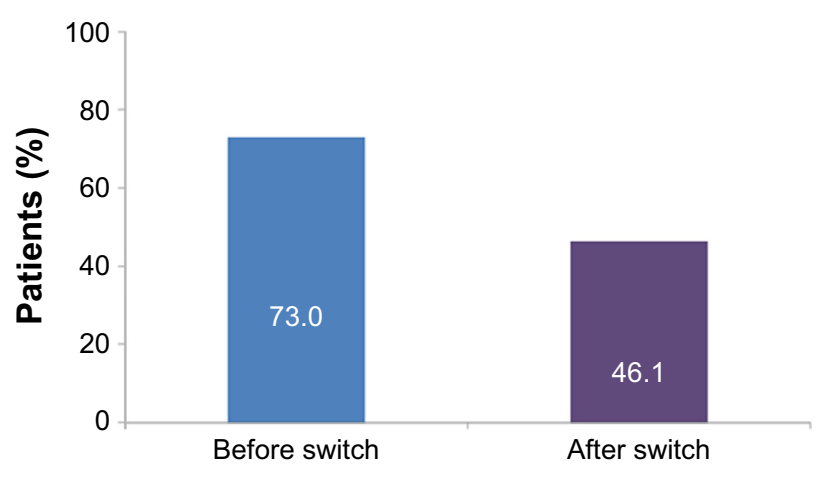

Figure 5 Percentage of patients with SPK after fluorescein staining before and after the treatment switch.

Note: The decrease was statistically significant $(P \leq 0.00$ I).

Abbreviation: SPK, superficial punctate keratitis.

In other words, switching from a preserved AT to an unpreserved one could lead to a quick improvement in OSDI and SPK frequency. This clinical result could support the findings of Meloni et al ${ }^{19}$ in preclinical assays.

A high correlation between SPK and high OSDI score has also been observed, before as well as after treatment switch. It is reasonable to assume that patients with a high OSDI score are highly likely to present also an SPK.

Overall, high OSDI scores and the frequency of SPK have significantly decreased (97 and 37\%, respectively, both $P \leq 0.001$ ) when switching to a preservative-free AT, although in some cases, the OSDI scores may remain high after treatment switch. One of the reason for this may be that recovery from ocular surface diseases may take longer in these patients after preservatives have been eliminated, confirming the sustained impact of preservatives on the eye surface. ${ }^{21-24}$ These data also confirmed that ATs, regardless the type of preservative, cause long-term damage to the eye surface and hence should be avoided in patients suffering from chronic DED, according to Jones et al. ${ }^{14}$

When asked for their preference, more than nine of the 10 patients preferred the preservative-free AT over their previous ATs.

We acknowledge that the above observations are not based on data collected through a comparative study, hence not allowing an intergroup comparison. Other weakness of this survey is the absence of data about concomitant pathologies or concomitant treatments, which both potentially may have impacted on their DED. Moreover, the survey did not plan for assessing the improvement in OSDI scores and frequency of SPK after the treatment switch. Despite these limitations, the present real-life survey allowed to collection of epidemiological data from a large patient sample providing an overview of the population suffering from DED in
Mexico and showing that severe DED and SPK are strongly present and sustained in patients using preserved ATs over a long time period to treat their disease. In addition, our survey showed that, when switching to a preservative-free AT, DED and SPK improve significantly, thereby confirming the clinical benefit of a preservative-free AT..$^{25,26}$

\section{Conclusion}

This survey confirmed that using preserved ATs, regardless the type of preservative, causes harm to the ocular surface even in case of short periods of treatment and that so-called "soft preservative" could be even more harmful to the ocular surface than traditional preservatives including BAK. Although the existence of concomitant pathologies or treatments have not been assessed, it also confirms that switching to preservative-free ATs improve markedly and rapidly the ocular surface status, with the result of a strong preference of the patients for the unpreserved treatment.

\section{Acknowledgments}

The authors acknowledge the participation of the members of the Grupo de Evaluación sobre Conservadores en Oftalmologia (GRECO) study group for the patient recruitment, follow-up, and collection of data. This survey was funded by Laboratories Théa, Mexico.

\section{Disclosure}

MR is a medical manager at Laboratories Thea Mexico. The authors report no other conflicts of interest in this work.

\section{References}

1. Craig JP, Nichols KK, Akpek EK, et al. TFOS DEWS II definition and classification report. Ocul Surf. 2017;15(3):276-283.

2. Stern ME, Schaumburg CS, Pflugfelder SC. Dry eye as a mucosal autoimmune disease. Int Rev Immunol. 2013;32(1):19-41.

3. Stevenson W, Chauhan SK, Dana R. Dry eye disease: an immune-mediated ocular surface disorder. Arch Ophthalmol. 2012;130(1):90-100.

4. Messmer EM. The pathophysiology, diagnosis, and treatment of dry eye disease. Dtsch Arztebl Int. 2015;112(5):71-81.

5. Chia EM, Mitchell P, Rochtchina E, Lee AJ, Maroun R, Wang JJ. Prevalence and associations of dry eye syndrome in an older population: the Blue Mountains Eye Study. Clin Experiment Ophthalmol. 2003;31(3): 229-232.

6. Schaumberg DA, Sullivan DA, Buring JE, Dana MR. Prevalence of dry eye syndrome among US women. Am J Ophthalmol. 2003;136(2): 318-326.

7. Lin PY, Tsai SY, Cheng CY, Liu JH, Chou P, Hsu WM. Prevalence of dry eye among an elderly Chinese population in Taiwan: the Shihpai Eye Study. Ophthalmology. 2003;110(6):1096-1101.

8. McCarty CA, Bansal AK, Livingston PM, Stanislavsky YL, Taylor HR. The epidemiology of dry eye in Melbourne. Aust Ophthalmol. 1998; 105(6):1114-1119.

9. Moss SE, Klein R, Klein BE. Prevalence of and risk factors for dry eye syndrome. Arch Ophthalmol. 2000;118(9):1264-1268. 
10. Schein OD, Hochberg MC, Munoz B, et al. Dry eye and dry mouth in the elderly: a population-based assessment. Arch Intern Med. 1999; 159(12):1359-1363.

11. Schiffman RM, Christianson MD, Jacobsen G, Hirsch JD, Reis BL. Reliability and validity of the Ocular Surface Disease Index. Arch Ophthalmol. 2000;118(5):615-621.

12. Dry Eye Workshop. The definition and classification of dry eye disease: report of the definition and classification subcommittee of the international dry eye workshop. Ocul Surf. 2007;5(2):75-92.

13. Downie LE, Keller PR. A pragmatic approach to the management of dry eye disease: evidence into practice. Optom Vis Sci. 2015;92(9): 957-966.

14. Jones L, Downie LE, Korb D, et al. TFOS DEWS II management and therapy report. Ocul Surf. 2017;15(3):575-628.

15. Barabino S, Rolando M, Nardi M, Bonini S, Aragona P, Traverso CE. The effect of an artificial tear combining hyaluronic acid and tamarind seeds polysaccharide in patients with moderate dry eye syndrome: a new treatment for dry eye. Eur J Ophthalmol. 2014;24(2):173-178.

16. Cohen S, Martin A, Sall K. Evaluation of clinical outcomes in patients with dry eye disease using lubricant eye drops containing polyethylene glycol or carboxymethylcellulose. Clin Ophthalmol. 2014;8: 157-164.

17. Diaz-Valle D, Arriola-Villalobos P, Garcia-Vidal SE, et al. Effect of lubricating eyedrops on ocular light scattering as a measure of vision quality in patients with dry eye. J Cataract Refract Surg. 2012;38(7): 1192-1197.

18. Doughty MJ. Fluorescein-tear breakup time as an assessment of efficacy of tear replacement therapy in dry eye patients: a systematic review and meta-analysis. Ocul Surf. 2014;12(2):100-111.
19. Meloni M, Pauly A, Servi BD, Varlet BL, Baudouin C. Occludin gene expression as an early in vitro sign for mild eye irritation assessment. Toxicol In Vitro. 2010;24(1):276-285.

20. Pauly A, Brasnu E, Riancho L, Bringole-Baudouin F, Badouin C. Multiple endpoint analysis of BAC-preserved and unpreserved antiallergic eye drops on a 3D-reconstituted corneal epithelial model. Mol Vis. 2011;17:745-755.

21. Baudouin C, Labbe A, Liang H, Pauly A, Brignole-Baudouin F. Preservatives in eyedrops: the good, the bad and the ugly. Prog Retin Eye Res. 2010;29(4):312-334.

22. Palmer RM, Kaufman HE. Tear film, pharmacology of eye drops, and toxicity. Curr Opin Ophthalmol. 1995;6(4):11-16.

23. Liu X, Yu FF, Zhong YM, Guo XX, Mao Z. Therapeutic effects of sodium hyaluronate on ocular surface damage induced by benzalkonium chloride preserved anti-glaucoma medications. Chin Med J. 2015; 128(18):2444-2449.

24. Mencucci R, Boccalini C, Caputo R, Favuzza E. Effect of a hyaluronic acid and carboxymethylcellulose ophthalmic solution on ocular comfort and tear-film instability after cataract surgery. J Cataract Refract Surg. 2015;41(8):1699-1704.

25. Perenyi K, Dienes L, Kornafeld A, et al. The effect of tear supplementation with $0.15 \%$ preservative-free zinc-hyaluronate on ocular surface sensations in patients with dry eye. J Ocul Pharmacol Ther. 2017;33(6): $487-492$.

26. Brjesky VV, Maychuk YF, Petrayevsky AV, Nagorsky PG. Use of preservative-free hyaluronic acid (Hylabak $\left.\left({ }^{\circledR}\right)\right)$ for a range of patients with dry eye syndrome: experience in Russia. Clin Ophthalmol. 2014; 8:1169-1177.
Clinical Ophthalmology

\section{Publish your work in this journal}

Clinical Ophthalmology is an international, peer-reviewed journal covering all subspecialties within ophthalmology. Key topics include: Optometry; Visual science; Pharmacology and drug therapy in eye diseases; Basic Sciences; Primary and Secondary eye care; Patient Safety and Quality of Care Improvements. This journal is indexed on

Submit your manuscript here: http://www.dovepress.com/clinical-ophthalmology-journal

\section{Dovepress}

PubMed Central and CAS, and is the official journal of The Society of Clinical Ophthalmology (SCO). The manuscript management system is completely online and includes a very quick and fair peer-review system, which is all easy to use. Visit http://www.dovepress.com/ testimonials.php to read real quotes from published authors. 